\title{
Analysis on the Characteristics and the Causes of Spatial-temporal Evolution of Population Distribution in Heilongjiang Province*
}

\author{
Chao Ni \\ Geography Department \\ Harbin University \\ Harbin, China
}

\author{
Xiangli Wu \\ Geography Department \\ Harbin Normal University \\ Harbin, China
}

\begin{abstract}
In order to analyze in depth spatial distribution characteristics of population in Heilongjiang Province and further master the regularity of population distribution, this paper took 13 prefecture-level cities in Heilongjiang Province as the subject investigated, and used the relative change rate of population density, structure index of population distribution, population center of gravity as methods to research spatialtemporal characteristics and variation trend of population distribution in Heilongjiang Province from 1986 to 2016. The results showed that: The population of Heilongjiang Province during the study period was steadily increasing, but thirteen areas belonging to Heilongjiang Province were distinctly different because of the differences in geographical location, economy, resources and other factors; the population density of Heilongjiang Province showed a trend of steady increasing in time, in space population density in southwest region was largest, followed by the eastern region and northwest region was the smallest. Population distribution of Heilongjiang Province in 1986 to 1996 was more balanced, later population gradually migrated to developed areas; the changing trajectory of population gravity center had certain regularity which was gradually moving from the northeast to the southwest.
\end{abstract}

Keywords-population distribution; spatial-temporal evolution; Heilongjiang Province

\section{INTRODUCTION}

Population is the basis and main body of all social production behaviors (Karl Marx, 1979), its content is complex and comprehensive social relations, which has a greater impact on economic and social development (Zhibin Zhang et al, 2013). Population distribution is a manifestation of the demographic process in a geographical space within a certain period of time (Huanyong $\mathrm{Hu} \&$ Shanyu Zhang, 1984), which is constrained by natural conditions, social production methods and economic development levels (Jian Yang et al, 2010). Population geography focuses on the spatial differences between population distribution and population change in different regions (Zhiming Feng \& Peng Li, 2011), and the study of population spatial distribution pattern and spatial-temporal evolution as the core issue of population geography has always been a hot

*Project: The National Social Science Fund of China (Project Number: 16BJY039). topic in academic research (Qiang Yang et al, 2016).

As early as the beginning of the 19th century, Humboldt and Ritter, the founders of modern geography, discussed the relationship between population size and distribution and their natural conditions such as topography and climate (Zhiming Feng \& Peng Li, 2011). The British epidemiologist Snow used the mapping method to reveal the spatial distribution of the population during the cholera in London in 1854 (Qi Feng \& Jinling Tang, 2017). The German human geographer Ratzel discussed population size, population distribution, population movement and the effects in the first geography writings of population distribution, which is entitled "Human Geography"(Jiqin Chen, 1987). French geographer Blache discussed the phenomenon of world population distribution and its causes in the book "Distribution of the World Population" and "Principles of Human Geography"(Jiqin Chen, 1987).

Domestic research on population distribution began in the 1920s and 1930s. In 1926, the founder of Chinese modern geography, Kezhen Zhu, published the first population geography paper "On the Population Density of Jiangsu and Zhejiang Provinces", which opened the study of population geography in China (Kezhen Zhu, 1999). In 1935, geographer Hu Huanyong proposed the "AiHui-TengChong" line in the writing "The Distribution of Chinese Population", revealing the disparity in the distribution of Chinese population, which is of great significance in population geography (Huanyong $\mathrm{Hu}, 1935$ ).

In recent years, some scholars have relevant studies on the spatial distribution of population in Heilongjiang Province. The demographic changes in Heilongjiang Province from the aspects of population density, age structure and health culture level were analyzed (Shaowei Liu, 1989). The characteristics of population development in Heilongiiang Province in the three decades of reform and opening up through indicators such as the total population were analyzed (Zhida Liu,2008). The evolution of Heilongjiang population between1949 and 2000 from the perspective of changes in nature and social structure was analyzed (Yanzhen Huang, 2008). Population distribution and the reasons for change in Heilongjiang Province in 2005 
was analyzed, which population density and other indicators was used (Lei Sun, 2008). According to characteristics of population distribution in Heilongjiang Province in 2008, population development functional areas were classified and scientifically guided (Shengzhong Lin, 2009). Based on the census data, the new trends and new changes in population development in Heilongjiang Province in 2010 were studied (Shusheng Liu, 2012; Li Zhang \& Zhida Liu, 2012). The coordinated development of population distribution and economic pattern in Heilongjiang Province was researched with the indexes, such as population and economic geography concentration (Xiangli Wu \& Haiyan Zhuang, 2014). The evolution of the spatial and temporal pattern of population in Heilongjiang Province in 2000 and 2010 was analyzed (Haiyan Zhuang \& Xiangli Wu, 2014).

However, the above studies have the following limitations. Some researches only studied the spatial distribution of population in Heilongjiang Province in a certain year or two, which lacked the spatial evolution characteristics for a certain period of time. Some researches only mentioned the time study of population distribution in Heilongjiang Province lacking spatial change analysis of population distribution. Other researches focused on the year of 2000 or 2010 , the study on the spatial distribution of population in Heilongjiang Province at the present stage was much less.

This paper analyzes the characteristics of population distribution in Heilongjiang Province from 1986 to 2016 by using the methods, such as relative change rate of population density, population distribution structure, population center of gravity and so on, which is not only of great significance for formulating the policy of optimizing population distribution and promoting the rational redistribution of population, but also plays a positive role in promoting the coordinated development of population and social and economic development in Heilongjiang Province.

\section{DATA AND METHODS}

\section{A. Data Sources}

This paper takes 12 prefecture-level cities such as Harbin and the Daxing'anling area under the jurisdiction of Heilongjiang Province as the basic research unit. The basic data mainly comes from the Heilongjiang Statistical Yearbook from 1987 to 2017. Considering the change of administrative divisions in Heilongjiang Province during the research period, in order to facilitate statistical analysis of data, based on the results of administrative divisions in 2014, this paper collates the population data of other years.

\section{B. Research Methods}

1) Relative change rate of population density: Population density refers to the degree of population density per unit area of land, and revealing the regional characteristics of population change can be represented by the relative change rate of population density. Its calculation formula is as follows (Lu Wang, 2014).

$$
F=\left(d_{i+1}-d_{i}\right) /\left(D_{i+1}-D_{i}\right)
$$

In the formula (1): $\mathrm{F}$ is the relative change rate of population density in the research unit $i ; d_{i}+1$ is the population density of the research unit $i$ at the end of the study; di is the population density of the research unit $i$ in the early stage of the study; $D_{i}+1$ is the national population density at the end of the study; $D_{i}$ is the national population density in the early stage of the study. $F<-1$ indicates the population density decreases rapidly; $-1 \leq F<0$ indicates the population density decreases slowly; $0 \leq F \leq 1$ indicates the population density increases slowly; $F>1$ indicates the population density increases rapidly.

2) Population distribution structure index: The commonly used indicator reflecting the concentration or dispersion of regional population distribution is the population distribution structure index, which can be expressed by the population distribution imbalance index and the concentration index. The calculation method is as follows (Fei Su \& Pingyu Zhang, 2010):

$$
\begin{aligned}
& U=\sqrt{\frac{\sum_{i=1}^{n} \frac{\sqrt{2}}{2}\left(X_{i}-Y_{i}\right)^{2}}{n}} \\
& C=\frac{1}{2} \sum_{i=1}^{n}\left|X_{i}-Y_{i}\right|
\end{aligned}
$$

In the formula (2) and (3): $\mathrm{U}$ is the unbalanced index; $C$ is the concentration index; $N$ is the number of study areas; $X_{i}$ is the ratio of the total population of the research unit $i$ to the total population of the research area; $Y_{i}$ is the ratio of the land area of study unit $i$ to the total land area of study area. The smaller the values of $U$ and $C$, the more balanced the population distribution is; on the contrary, the more concentrated the population distribution is.

3) Population gravity center: The concept of population gravity center was firstly put forward by Francis Walker in 1874 , which was an important index to measure the overall trend and activity of population distribution. It was generally showed in latitude and longitude of maps (Qiang Yang et al, 2016; Fei Su \& Pingyu Zhang, 2010):

$$
\begin{aligned}
& X=\sum_{i=1}^{n} P_{i} X_{i} / \sum_{i=1}^{n} P_{i} \\
& Y=\sum_{i=1}^{n} P_{i} Y_{i} / \sum_{i=1}^{n} Y_{i}
\end{aligned}
$$

In the forma (4): $X$ and $Y$ are the longitude and latitude of the population gravity center in the study area respectively; $N$ is the number of statistical units in the study area; $P_{i}$ is the 
population of statistical unit; $X_{i}$ and $Y_{i}$ are the longitude and latitude of the center of statistical unit i respectively.

\section{RESULTS AND ANALYSIS}

\section{A. The Population has Steadily Increased, But Has Great Difference in the Regional Distribution}

During the study period, the population of Heilongjiang Province has risen, from 33.32 million in 1986 to 36.59 million in 2016, and the population increased by 3.27 million in 31 years. Baby boom led to the rapid growth of population in Heilongjiang Province from 1986 to 2000, the population has increased 4.76 million during 15 years, with an average annual growth rate of about $0.96 \%$, which is about 3.06 times that of the whole research period. From 2000 to 2012, the population growth rate of Heilongjiang Province gradually slowed down, increasing only 27 million in 13 years, with an average annual increase of less than $0.06 \%$. Influenced by low birth rate and net population migration, the population of Heilongjiang Province declined rapidly from 2012 to 2016. In a short period of five years, the population decreased about 174.99 million, resulting in negative population growth.

However, the population in Heilongjiang Province from 1986 to 2016 showed great regional differences. During the study period, the population of Harbin greatly increased, from 3.86 million in 1986 to 9.62 million in 2016, with an increase of 5.76 million in 31 years. The population in Daqing, Shuangyashan and Jixi increased less than in Harbin. The population growth trend was relatively flat, such as Hegang, Qitaihe, Heihe and Daxing'anling. Population of Jiamusi decreased most obviously, from 3.74 million in 1986 to 2.29 million in 2016. The population of Mudanjiang, Suihua and Qiqihar decreased 0.53 million, 0.42 million and 0.25 million respectively. While the lowest population decline was in Yichun, the number was only 0.09 million.

\section{B. Population density was growing slowly, while some cities' were above the national average}

Compared with the increasing population density of China, the population density in Heilongjiang Province increased first and then decreased, from $73.62 \mathrm{p} / \mathrm{km}^{2}$ in 1986 to $84.72 \mathrm{p} / \mathrm{km}^{2}$ in 2012 , and then the population density decreased to $80.86 \mathrm{p} / \mathrm{km}^{2}$ in 2016 due to the reduction of population.

Generally speaking, cities which population density increased included Harbin, Qitaihe, Daqing, Shuangyashan, Jixi, Hegang, Daxing'anling and Heihe.

Under the influence of the attractiveness of provincial capital cities, Harbin's population density has been greater than the population density value of Heilongjiang Province in the same year since 1991, and it has been greater than the national population density value since 1997 .

As the largest coking coal production base in Northeast China, the population in Qitaihe is relatively dense, and the population density from 1986 to 2016 has always been greater than the population density of Heilongjiang Province.
Due to the concentration of the petrochemical industry on the population, Daqing which was known as the capital of green oil has had a significantly higher population density since 1993 than the same period in Heilongjiang Province. The population density curves of Shuangyashan, Jixi and Hegang are similar, which are slightly lower than the population density values in Heilongjiang Province during the same period. The curves increased rapidly at first and then came to flat. The trend of increasing population density in Daxing'anling and Heihe is extremely weak and the population density of Jiamusi, Mudanjiang, Suihua, Qiqihar and Yichun decreased from 1986 to 2016.

The spatial distribution characteristic of population density in Heilongjiang Province was as follows: the population density in southwest Province is larger, followed by that in east Province, and is smaller in northwest Province. A conclusion can be draw from the four years of 1986, 1996, 2006 and 2016 (as shown in "Fig. 1", "Fig. 2", "Fig. 3" and "Fig. 4"): Core areas of population density in Heilongjiang Province were Harbin and Suihua. The increase of population density in Harbin, which was the capital city of Heilongjiang Province, was greatest during the study period from $72.74 \mathrm{p} / \mathrm{km}^{2}$ to $181.26 \mathrm{p} / \mathrm{km}^{2}$. It was significantly higher than that of Heilongjiang Province. The population density of Suihua reached $167.75 \mathrm{p} / \mathrm{km}^{2}$ in 1986 , but it has been declining in recent years. Because of abundant petroleum resources, the population density of Daqing increased rapidly. Although the population density was sparse at the beginning of the study, now it has leaped into the high population density area of Heilongjiang Province. The population density of Qitaihe and Qiqihar is relatively stable, and has been in a high population density zone during the study period. The population density of Jixi, Shuangyashan and Hegang increased significantly, while that of Mudanjiang and Jiamusi decreased significantly. These areas belong to the middle and low population density areas of Heilongjiang Province. Daxing'anling, Heihe and Yichun are sparse areas of population density in Heilongjiang Province. Especially, the population distribution of Daxing'anling is less than $9 \mathrm{p} / \mathrm{km}^{2}$, which is the sparsest area in Heilongjiang Province. This mainly related to its geographical location. Because of the development of border trade, the population density of Heihe was growing slowly and slightly higher than that of Daxing'anling. As the important forestry production base in China, the population density of Yichun was less than half of the average level of Heilongjiang Province. 


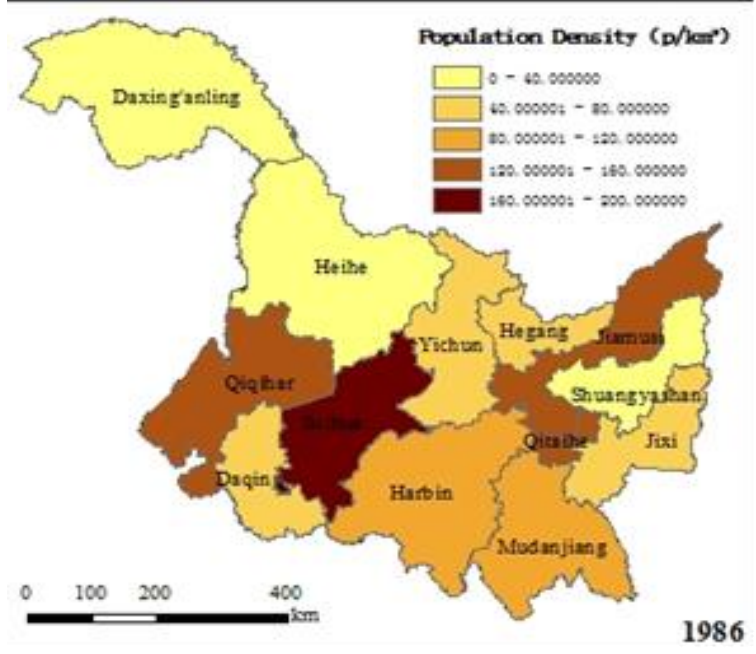

Fig. 1. Population Density of Heilongjiang Province in 1986.

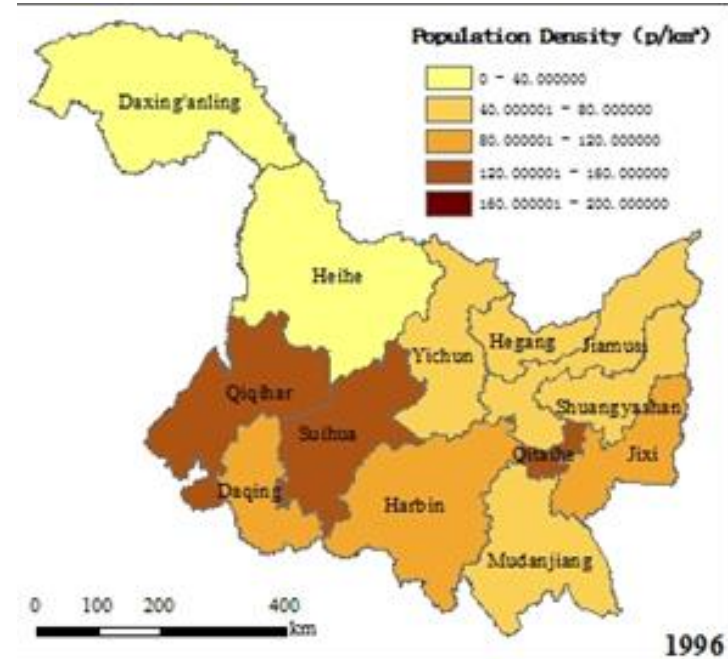

Fig. 2. Population Density of Heilongjiang Province in 1996.

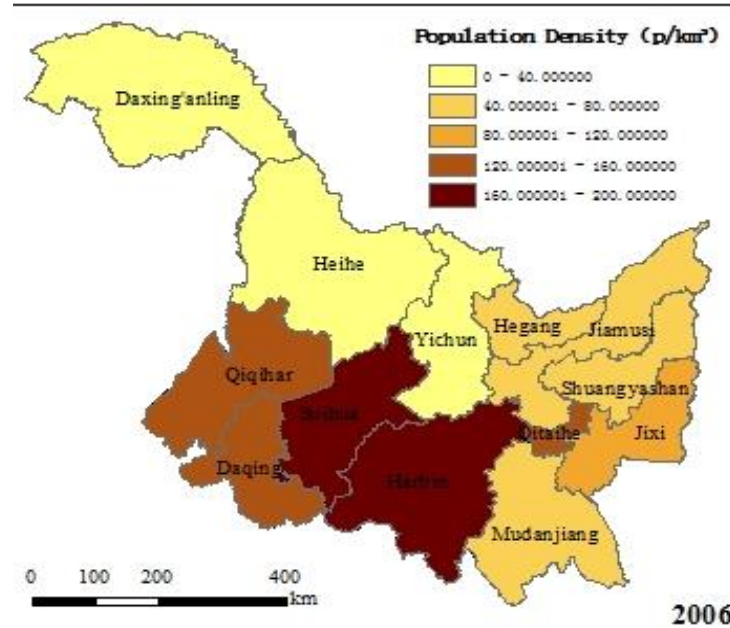

Fig. 3. Population Density of Heilongjiang Province in 2006.

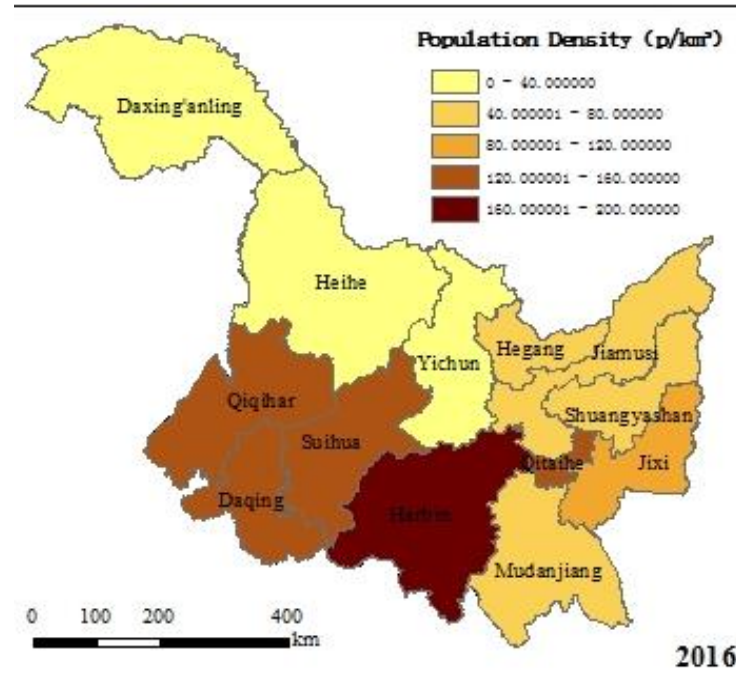

Fig. 4. Population Density of Heilongjiang Province in 2016.

The trend chart shown in "Fig. 5", the relative change rate of population density of Heilongjiang Province was 0.23 which meant a slow increase in population density, However there were significant differences among each research unit. The relative change rate of population density in Harbin, Daqing and Shuangyashan is more than one, which showed the population density increased rapidly. While the relative change rate of population density in Daxing'anling, Heihe, Qitaihe, Hegang and Jixi was less than or equal to one, it showed that the population density in these areas has increased slowly. At the beginning of the study, the population density of Jiamusi was $115.28 \mathrm{p} / \mathrm{km}^{2}$, and at the end of the study, it reduced to $70.55 \mathrm{p} / \mathrm{km}^{2}$, which made the population density decreasing rapidly and the relative change rate being less than minus one. The relative change rate of population density was still negative value, which showed the decreased trend in Suihua, Qiqihaer, Yichun and Mudanjiang was relatively slow. 


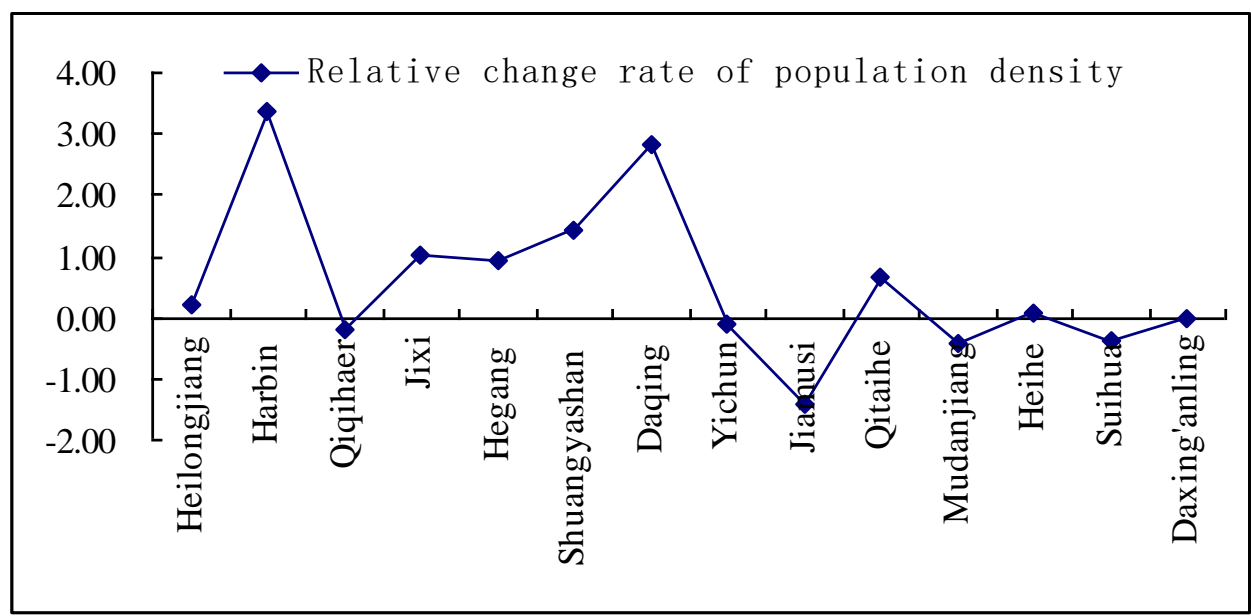

Fig. 5. Relative change rate of population density of Heilongjiang Province in 1986-2016.

\section{Population Distribution Was Imbalanced and Phenomenon of Agglomeration to Big Cities Was Obvious}

As can be seen from "Fig. 6", population equilibrium index of Heilongjiang Province showed a slight growth trend from 1986 to 2016 . From 0.051 at the beginning of the study to 0.057 at the end of the study, the average annual growth rate is about $0.37 \%$. Population distribution shows a trend of relative agglomeration. According to the changing characteristics of the curve, it was divided into two periods:

The population unbalance index continued to decrease from 0.051 to 0.045 , which indicated that the population distribution in all regions of Heilongjiang Province was comparative equilibrium from 1986 to 1996.

The changing trend of population unbalance index increased obviously from 1996 to 2016, and the average annual growth rate was $1.21 \%$. The trend of population distribution concentrating to local area increased, affecting by the level of social and economic development. The population increased rapidly in Harbin and Daqing City, because of developed economy.

The trend of population concentration index in Heilongjiang Province is similar to that of population imbalance index. Although it reduced to the minimum in 1996, the curve showed an increasing trend. During the study period, it increased from 0.292 to 0.312 . It showed that the population distribution in Heilongjiang Province was gradually relatively concentrated. The main reason was economic disparity. Cities, such as Harbin, Daqing and so on, had economic advantages; their ability to agglomerate the population had continuously strengthened which attracting people moving to more developed areas.

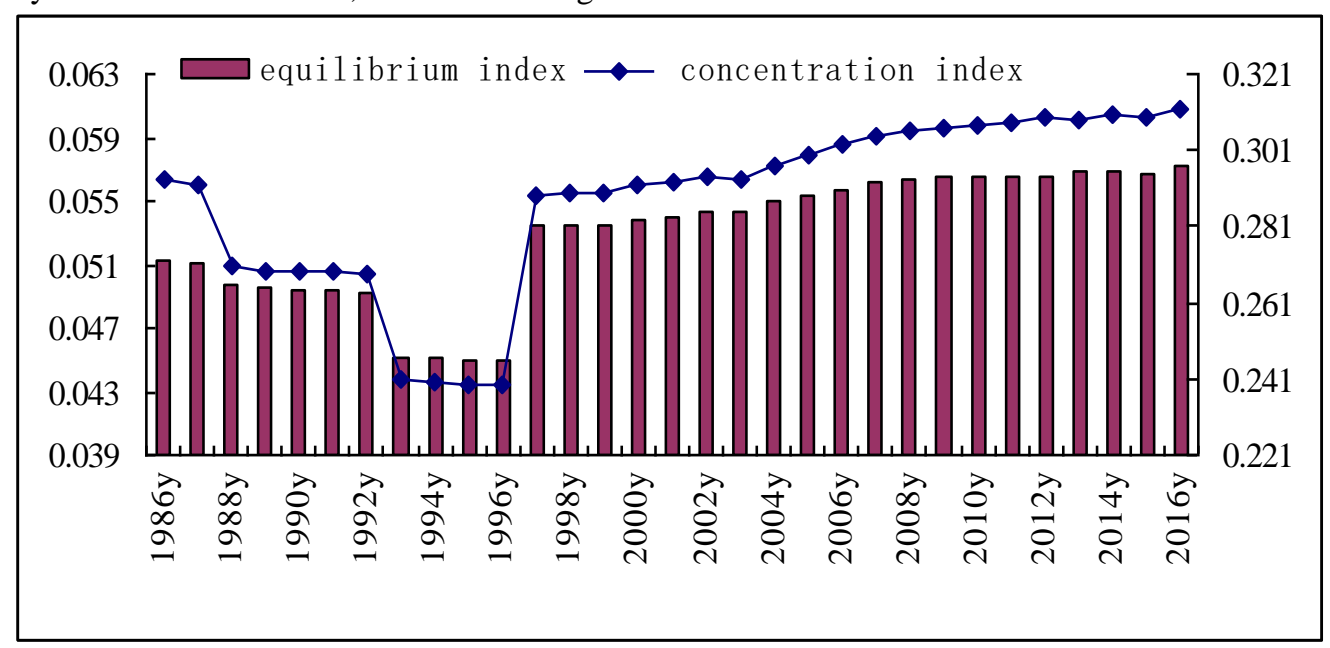

Fig. 6. Population equilibrium index and concentration index of Heilongjiang Province in 1986-2016. 


\section{Population Gravity Center Moved Obviously from the Northeast to the Southwest}

The movement of population gravity center in Heilongjiang Province has presented certain regularity since 1986, which showed a trend of gradual movement from northeast to southwest. According to the agglomeration degree of distribution of population gravity center, it was divided into three periods: (1) Population gravity center in Heilongjiang Province was located in Qing'an County of Suihua City from 1986 to 1990, which moved from the Southwest to the Northeast, and the longitude was $127.419^{\circ}$ $127.441^{\circ} \mathrm{E}$ and the latitude was $46.702^{\circ}-46.706^{\circ} \mathrm{N}$. (2) Population gravity center in Heilongjiang Province was located in the junction area of Suihua City and Harbin City from 1991 to 1996, population gravity center moved to Bayan County in 1991, but it was located in Qing'an County in the rest years. Population gravity center moved from Southwest to Northeast, but the movement was relatively small, which moved toward east in longitude and north in latitude. (3) Population gravity center was located in Bayan County from 1997 to 2016, and moved from Northeast $\left(127.311^{\circ} \mathrm{E}, 46.580^{\circ} \mathrm{N}\right)$ to Southwest $\left(127.247^{\circ} \mathrm{E}, 46.561^{\circ} \mathrm{N}\right)$, namely it moved westward by $0.064^{\circ}$ in longitude and southward by $0.019^{\circ}$ in latitude.

Population gravity center in Heilongjiang Province moved from Suihua City to Harbin City gradually during the research period, which moved westward by $0.172^{\circ}$ in longitude and southward by $0.141^{\circ}$ in latitude. As the center of Northeast Asia, Harbin was the political, economic and cultural center of Heilongjiang province. Harbin was the megalopolis in Heilongjiang Province with a large population, the attraction of city made the population gravity center moving to Southwest.

\section{DISCUSSION}

The characteristics of spatial and temporal distribution of population in Heilongjiang Province were analyzed with the relative change rate of population density, population distribution structure index and population center of gravity. Comparing with current research of using single indicators, various methods were used and the change of population spatial pattern in Heilongjiang Province could be reflected more accurately.

Continuous change was the characteristics of population distribution; current research could not accurately reflect the dynamic process of population change in Heilongjiang Province with the data for only one or two years. The research period was from 1986 to 2016 and the data for 31 years was used. The timing continuity of population distribution could reflect the dynamic characteristics of population in Heilongjiang Province compared with the existing research results.

Population distribution was an extremely complex question. The characteristics of population quantity and spatial distribution in each city (region) of Heilongjiang Province were analyzed preliminarily. In order to discover the temporal and spatial distribution trend of population and its regularity better in future studies, the formation mechanism of spatial pattern of population distribution will be discussed in depth from a smaller spatial unit.

\section{CONCLUSION}

Studying the evolution characteristics of population distribution in Heilongjiang Province was of great practical significance for grasping the spatial distribution law of population, recognizing the characteristics of regional population growth, formulating corresponding population policies and realizing regional economic development. This paper studied the spatial and temporal characteristics of population distribution in 13 research units of Heilongjiang Province from 1986 to 2016, and came to the following conclusions:

Population in Heilongjiang Province increased steadily from 1986 to 2016, the tendency to population decline which was affected by many factors, such as low birth rate, population migration and so on, was obvious in recent years and population change has major variability in different cities.

The population density of Heilongjiang Province gradually increased during the past 31 years; the fluctuation range was small and has been lower than the national average. However the population density of mega-cities with faster economic development increased rapidly, far exceeding the national average population density.

The population equilibrium index and concentration index of Heilongjiang Province steadily decreased from 1986 to 1996, but they increased during the study period. Population distribution gradually gathered in some areas with better economic environment, such as Harbin and Daqing.

Because of the influence of economic status and location advantages, the motion trail of population gravity center in Heilongjiang Province has its regularity since 1986, which gradually moved from northeast to southwest, namely it gradually moved from Qing'an County of Suihua to Bayan county of Harbin in administrative area.

\section{REFERENCES}

[1] Karl Marx, Frederick Engels. Works by Karl Marx and Frederick Engels(Volume 46) [M].Beijing:People's Publishing House, 1979:37.

[2] Zhang Zhibin, Pan Jing, Li Xiaohu. The Spatial Evolution and Formation Mechanism of Population Density in Lanzhou City Over the Past 30 Years [J].Scientia Geographica Sinica, 2013, 3(1):36-44.

[3] Hu Huanyong, Zhang shanyu. Population Geography of China [M] Shanghai: East China Normal University Press, 1984:196.

[4] Yang jian, Pu Yingxia, Qin Xianghong,etc. The Spatial Distribution Pattern of Population and Its Analysis of the Spatio-Temporal Dynamics in Zhejiang Province [J]. China Population, Resources and Environment, 2010 ,20(3):95-99.

[5] Feng Zhiming, Li Peng.Review of Population Geography in the Past Century [J]. Progress in Geography, 2011, 30(2):131-140.

[6] Yang Qiang, Li Li,Wang Yundong,etc. Spatial Distribution Pattern of Population and Characteristics of its Evolution in China during 19352010 [J]. Geographical Research, 2016, 35(8): 1547-1560. 
[7] Feng Qi, Tang Jinling. Study on the 19th century Puerperal Fever Epidemic:Semmelweis-an often Neglected Pioneer Epidemiologist [J]. Chinese journal of Epidemiology, 2017, 38(8): 1136-1139.

[8] Chen Jiqing. Process in Research on Population Geography[J].Scientia Geographics Sinica, 1987, 7(2): 187-192.

[9] Zhu Kezhen. Literary Record of Zhu Kezhen [M].ZheJiang: ZheJiang Literature\&Art Publishing House, 1999:203-211.

[10] Hu Huanyong. Population Distribution in China [J].Acta Geographica Sinica, 1935, 2(1): 33-74.

[11] Liu Shaowei, Zhang Shuwei.Fourty years of population change in Heilongjiang Province [J].Population science of China, 1989(5): 4953.

[12] Liu Zhida. Analysis on the Characteristics of Heilongjiang Province's Population Development in the Past 30 Years [J]. Statistics and Consultation, 2008(5): 34-35.

[13] Huang Yanzhen. Analysis on the Evolution of Heilongjiang Population after liberation [J]. Heilongjiang Chronicles, 2008(1):8-9.

[14] Sun Lei. Analysis of the Population Distribution and its Change Reasons in Heilongjiang Province [J]. Population \& Economics, 2008(S1): 54-56.

[15] Lin Shengzhong, Zheng Weijun. Study on the Population Distribution in Heilongiiang Province [J]. Population Journal, 2009(5): 17-22.

[16] Liu Shusheng. New Trends and Changes of Population Development in Heilongjiang Province: A Study Based on Census Data [J]. Northern Economy and Trade, 2012(6): 3-4.

[17] Zhang Li, Liu Zhida.New trends and changes of population development in Heilongjiang Province [J]. Heihe Journal, 2012, 11: 181-182.

[18] Wu Xiangli, Zhuang Haiyan. Study on the Coordinative Development of Population Distribution and Pattern of the Economy in Heilongjiang Province [J]. Areal Research and Development, 2014, 33(1): 164-169.

[19] Zhuang Haiyan,Wu Xiangli. A Study on the Temporal and Spatial Evolution of Population in Heilongjiang Province [J]. Natural Science Journal of Harbin Normal University, 2014, 30(4): 115-120.

[20] Wang Lu, Feng Zhiming,Yang Yanzhao,etc al. The change of population density and its influencing factors from 2000 to 2010 in China on county scale [J]. Acta Geographica Sinica, 2014, 69(12): 1790-1798.

[21] Su Fei, Zhang Pingyu.Spatio-temporal Dynamics of Population Distribution in the Middle and Southern Liaoning Urban Agglomeration [J]. Progress in Geography, 2010, 29(1): 96-102. 